\title{
Commentary: "Alpha-enolase (ENO1) controls alpha v/beta 3 integrin expression and regulates pancreatic cancer adhesion, invasion, and metastasis"
}

\author{
Moitza Principe ${ }^{1,2}$, Simone Borgoni ${ }^{1,2}$, Francesco Novelli ${ }^{1,2,3}$ \\ 'Department of Molecular Biotechnology and Health Sciences, University of Turin, Italy \\ ${ }^{2}$ Center for Experimental Research and Medical Studies (CeRMS), Azienda Universitaria Ospedaliera Città della Salute e della Scienza di Torino, Italy \\ ${ }^{3}$ Molecular Biotechnology Center, University of Turin, Turin, Italy
}

Article Info

\section{Article Notes}

Received: August 10, 2017

Accepted: September 14, 2017

\section{*Correspondence:}

Dr. Francesco Novelli, Ph.D.

Center for Experimental Research and Clinical Studies (CeRMS), Azienda Ospedaliera Città della Salute e della Scienza di Torino, Via Santena 5, 10126 Turin, Italy Tel: +39-011-6336886 Fax: +39-011-6336887, Email: franco.novelli@unito.it

(c) 2017 Principe M. This article is distributed under the terms of the Creative Commons Attribution 4.0 International License.

\section{Keywords}

Pancreatic cancer

ENO1

Integrin

UPAR

Invasion

\section{ABSTRACT}

We have previously shown that in pancreatic ductal adenocarcinoma (PDA) cells, the glycolytic enzyme alpha-enolase (ENO1) also acts as a plasminogen receptor and promotes invasion and metastasis formation. Silencing of ENO1 in PDA cells induces oxidative stress, senescence and profoundly modifies PDA cell metabolism. Although anti-ENO1 antibodies inhibit PDA cell migration and invasion, little is known about the role of ENO1 in regulating cell-cell and cellmatrix contacts. We recently investigated the effect of ENO1 silencing on the proteome of PDA cells, and there was a significant downregulation of proteins involved in cell-cell and cell-matrix adhesion, including alphaV/beta3 integrin in ENO1-silenced PDA cells. These changes impaired the ability of ENO1silenced cells to adhere to collagen I and IV and fibronectin, and caused an increase in RGD (tripeptide Arg-Gly-Asp)-independent adhesion to vitronectin (VN) via urokinase plasminogen activator receptor (UPAR). Binding of UPAR to VN triggers integrin-mediated signals, which results in ERK1/2 and Rac activation, accumulation of ROS (Reactive Oxygen Species) and senescence. In ENO1-silenced cancer cells, the use of an anti-uPAR antibody led to reduced ROS production and senescence, and an increase in cell apoptosis. Overall, a decrease of in vitro and in vivo cell migration and invasion of ENO1silenced PDA cells was observed. This commentary summarizes new data demonstrating that ENO1 promotes PDA survival, migration and metastasis by cooperating with integrins and UPAR. These data represent a springboard for a novel therapeutic strategy to counteract PDA progression based on combined targeting of integrins, UPAR and ENO1.

\section{Background}

The involvement of alpha-enolase (ENO1) in cancer progression has become increasingly more addressed and described in recent years. Disease-related roles of EN01 are mostly attributed to its immunogenic capacity, DNA-binding ability and plasminogen receptor function ${ }^{1}$. In particular, ENO1 overexpression has been associated with cancer proliferation and invasion, and correlated with poor clinical outcomes ${ }^{2,3}$. The role of ENO1 in pancreatic ductal adenocarcinoma (PDA) has been reported in the literature by our previous studies; firstly we identified a correlation between EN01-circulating antibodies in the blood and a better response to therapy and survival of PDA patients ${ }^{4,5}$; we then defined EN01 as a tumor-associated antigen that induces both humoral and $\mathrm{T}$ cell-specific responses in PDA patients ${ }^{6}$; finally we found an association of ENO1 with the promotion of PDA tumor invasion due 
to its role as a plasminogen receptor, by promoting matrix degradation through plasminogen activation ${ }^{7}$. Moreover, we demonstrated that targeting EN01 using passive immunotherapy with anti-EN01 antibodies showed good results in terms of reducing metastasis and extending survival in vivo ${ }^{7}$. In addition, DNA-vaccination against EN01 in a mouse model obtained similar results ${ }^{8}$. Finally, EN01 has been found to be one of the leading regulators of the Warburg effect and plays an important role in carcinogenesis and tumor maintenance ${ }^{9}$. It is known that inhibition of glycolysis prevents cancer development as it is essential for proliferation, invasion and metastasis of cancer ${ }^{10}$. Analysis of silenced ENO1 in PDA cells revealed a profound modification in their metabolism, which was associated with an increase of oxidative stress and senescence ${ }^{9}$. Starting from this evidence, the following study was carried out to highlight the key role of EN01 in pancreatic cancer, with particular focus on the regulation of cell-cell and cell-matrix contacts and functions.

\section{Our results}

In the study "Alpha-Enolase (ENO1) controls alpha v/ beta 3 integrin expression and regulates pancreatic cancer adhesion, invasion, and metastasis"11, we characterized the role of ENO1 as a multifunctional protein involved in migration and invasion in PDA by exploiting a knockdown using shRNA. Using a combination of atomic force microscopy and confocal microscopy, we were able to observe strong morphological changes in shEN01 PDA cells, especially showing an increase in surface roughness due to the loss of cytoskeleton organization. The reorganization of cytoskeleton proteins suggested that the absence of ENO1 caused impairment of the ability of PDA cells to adhere to the ECM (extracellular matrix) and migrate.

Liquid chromatography- Tandem Mass Spectrometry (LC-MS/MS), a semi-quantitative proteomic analysis, was previously performed on EN01-silenced and control CFPAC-1 pancreatic cancer cells. Compared to control CFPAC-1 cells, EN01-silenced cells showed significantly altered expression of 17 proteins involved in cell adhesion and cytoskeleton organization, which was further confirmed by PCR analysis ${ }^{9}$. In particular, the proteins: actin-related protein 2/3 complex subunit 4 (ARPC4), F-actin-capping protein subunit alpha2 (CAPZA2) and breast cancer antiestrogen resistance protein 1 (BCAR1), usually associated with actin remodeling and poor prognosis in cancer ${ }^{12-15}$ were up-regulated after EN01 silencing. Paradoxically, the increase in these proteins was notassociated with increased aggressiveness of pancreatic cancer, as EN01-silenced cells showed a reduced ability to grow and invade both in vivo and in vitro. Further analysis of the post-transcriptional modifications of these proteins after EN01 silencing could help to clarify this discrepancy. Instead, other proteins generally associated with poor prognosis, such as Galectin
3 (LGALS3), family with sequence similarity 129 member B (FAM129B), integrin subunit alpha V (ITGAV) and MUC5AC (Mucin $5 A C)^{16-19}$ or those that augmented metastasis, such as Ahnak Nucleoprotein (AHNAK), Anterior Gradient 2 (AGR2), PDZ and LIM domain 2 (PDLIM1), Galectin 4 (LGALS4), Golgi Glycoprotein 1 (GLG1) and Serpin Family B Member 5(SERPINB5) ${ }^{20-25}$ were down-regulated in PDA cells. Of note, the interaction of some of these proteins with cytoskeleton elements is critical for cell shape modifications: PDLIM1 forms complexes with a-actinin1 and 4, thus regulating cell invasion and metastasis formation; LGALS3 interacts with $\beta$-catenin increasing tumorigenesis; and catenin delta 1 (CTNND1) interacts with E-cadherin promoting cell spreading ${ }^{26}$. Other down-regulated proteins were proposed as targets for PDA cancer therapy, such as ITGAV and AGR2, or as early biomarkers like MUC5AC and LGALS4 ${ }^{27,28}$. In accordance with the proteomic data ${ }^{9}$, we observed that silenced EN01 PDA cells showed a decreased ability to adhere to the ECM (with the exception of vitronectin $(\mathrm{VN})$, and a reduction in invasion and migration, suggesting a central role for ENO1 in metastasis spreading. Among all the proteins, we focused on integrins that are involved in the cell-ECM interaction. Silencing of ENO1 caused an increase of alpha5/beta1 and a decrease of alphaV/beta3 integrins. Fibronectin (FN) is recognized by both complexes, which cooperate in promoting cellular attachment and spreading ${ }^{29}$ with distinct roles: alpha5/ beta1 integrins determine adhesion strength through their catch-bond binding to FN, whereas alphaV/beta3 integrins mediate force-induced reinforcement of the adhesion site through their connection with the actin cytoskeleton ${ }^{30}$. We hypothesized that even in the presence of alpha5/beta1 integrins, which start the adhesive process, the absence of alphaV/beta3 in ENO1-silenced PDA cells impairs the reinforcement signals to establish strong contacts with the matrix and migrate. Interestingly, alphaV complexes with different subunits (beta1, beta3, beta5, beta6 and beta8) mediate adhesion to the most common ECM proteins, such as $\mathrm{FN}$, collagens (COLs) and $\mathrm{VN}^{31}$. Consequently, downregulation of alphaV in ENO1-silenced PDA cells is one of the major triggering factors for the decrease in their binding to ECM, with the exception of binding to $\mathrm{VN}$, which actually increases. In cancer, $\mathrm{VN}$ interacts with members of the integrin family (alphaV/beta1, alphaV/beta3, alphaV/ beta5 and alphaIIb/beta3) through the RGD motif ${ }^{32}$. As alphaV and beta3 subunits were down-regulated in ENO1silenced cells, all of the above-mentioned complexes cannot be considered responsible for the VN-increased adhesion of shEN01 cells; this led us to hypothesize that a non-integrin receptor is involved in the binding of VN. Urokinase Plasmin Activator Receptor (UPAR) binds the $\mathrm{N}$-terminal SMB domain on $\mathrm{VN}$ and, as the binding site is different from that integrins, usually the two groups of proteins bind VN independently, but cooperate for 
intracellular signaling ${ }^{32,33}$. uPAR lacks a cytosolic domain but is able to send downstream signals by associating with trans-membrane integrins (mainly beta 1 and beta3), even independently from direct integrin/matrix interaction in a ligand-independentmanner ${ }^{33}$. In this study, we demonstrate how, in the absence of ENO1, UPAR is up-regulated and is responsible for the strong interaction between cells and $\mathrm{VN}$, even in the absence of binding of integrins. Moreover, the absence of beta3 with a concomitant expression of beta1 integrin causes an unbalance in the intracellular signaling, leading to activation of proto-oncogene tyrosineprotein kinase $\mathrm{Src}$ and extracellular signal-regulated kinases (ERK1-2), and p38 mitogen-activated protein kinase (MAPK) inactivation. The ERK signal provided by UPAR-beta1 integrin interaction is required for activation of the small signaling GTPase subfamily $\mathrm{Rac}^{34}$. Taken together, these signals drive the cells to a senescence status, impairing tumor progression as well as apoptosis. Interestingly, we also found that, in the absence of ENO1, targeting of uPAR efficiently prevents ROS production and senescence while promoting apoptosis, suggesting that a combinatory strategy to simultaneously target ENO1 and UPAR could be effective to inhibit PDA tumor progression and invasion.

\section{Future perspectives}

Overall, we demonstrated a high dependency of PDA cells on components of the integrin family to exert their spreading capability. While we clearly showed that ENO1 is essential in mediating this effect, many other points remain to be addressed and investigated in future studies; a deeper investigation of additional targets identified from our proteomic screening could define other pathways dependent on ENO1. Moreover, from a clinical perspective, it is clear that ENO1 represents an optimal hit for targeted therapies. Particularly, the strong effects we demonstrated in reducing tumor spreading could be combined with other therapies targeting UPAR, inhibiting the intracellular signaling of integrin beta1/beta3, or inhibiting the binding between cells and ECM promoting cell death through anoikis. Additional investigations could include addressing transcription regulation mediated by ENO1, in order to unravel possible transcription factors correlated to the phenotypes seen in the knock-down cells. In this view, alterations in the signaling pathways in the cells could lead to epigenetic remodeling that is worth investigating in future studies.

\section{Acknowledgements}

We thank Dr. Radhika Srinivasan for helpful discussion and critical reading of the manuscript.

\section{Funding}

This study was supported by the Associazione Italiana
Ricerca sul Cancro (AIRC 5xmille no.12182 and IG no.15257); University of Turin-Progetti Ateneo 2014-Compagnia di San Paolo (PC-METAIMMUNOTHER), Italian Ministry of Health, Progetti Ricerca Finalizzata (RF-2013-02354892), Fondazione Ricerca Molinette Onlus, Fondazione Nadia Valsecchi Onlus and Fondazione Ursula e Giorgio Cytron.

\section{References}

1. Diaz Ramos A, Roig Borrellas A, Garcia Melero A, et al. alpha-Enolase, a multifunctional protein: its role on pathophysiological situations. J Biomed Biotechnol. 2012; 2012: 156795.

2. Capello M, Ferri Borgogno S, Cappello P, et al. alpha-Enolase: a promising therapeutic and diagnostic tumor target. FEBS J. 2011; 278: 1064-74.

3. Ceruti P, Principe M, Capello $M$, et al. Three are better than one: plasminogen receptors as cancer theranostic targets. ExpHematolOncol. 2013; 2: 12.

4. Tomaino B, Cappello P, Capello M, et al. Autoantibody signature in human ductal pancreatic adenocarcinoma. J Proteome Res. 2007; 6(10): 4025-4031.

5. Tomaino B, Cappello P, Capello M, et al. Circulating autoantibodies to phosphorylated alpha-enolase are a hallmark of pancreatic cancer. J Proteome Res. 2011; 10(1): 105-112.

6. Cappello P, Tomaino B, Chiarle R, et al. An integrated humoral and cellular response is elicited in pancreatic cancer by alpha-enolase, a novel pancreatic ductal adenocarcinoma adenocarcinoma-associated antigen. Int J Cancer. 2009; 125(3): 639-648.

7. Principe M, Ceruti P, Shih NY, et al. Targeting of surface alpha-enolase inhibits the invasiveness of pancreatic cancer cells. Oncotarget. 2015; 6(13): 11098-113.

8. Cappello P, Rolla S, Chiarle R, et al. Vaccination With EN01 DNA Prolongs Survival of Genetically Engineered Mice With Pancreatic Cancer. Gastroenterology. 2013; 144(5): 1098-1106.

9. Capello M, Ferri Borgogno S, Riganti C, et al. Targeting the Warburg effect in cancer cells through ENO1 knockdown rescues oxidative phosphorylation and induces growth arrest. Oncotarget. 2016; 7(5): 5598-612.

10. Heiden MGV. Targeting cancer metabolism: a therapeutic window opens. Nat Rev Drug Discov. 2011; 10: 671-84.

11. Principe M, Borgoni S, Cascione M, et al. Alpha-Enolase (ENO1) controls alpha $\mathrm{v} /$ beta 3 integrin expression and regulate pancreatic cancer adhesion, invasion, and metastasis. J HematolOncol. 2017; 10(1): 16.

12. Goley ED, Rammohan A, Znameroski EA, et al. An actin-filamentbinding interface on the Arp2/3 complex is critical for nucleation and branch stability. Proc Natl AcadSci U S A. 2010; 107: 8159-64.

13. Ou K, Yu K, Kesuma D, et al. Novel breast cancer biomarkers identified by integrative proteomic and gene expression mapping. J Proteome Res. 2008; 7: 1518-28.

14. Kumbrink J, Soni S, Laumbacher B, et al. Identification of Novel Crkassociated Substrate (p130Cas) Variants with Functionally Distinct Focal Adhesion Kinase Binding Activities. J Biol Chem. 2015; 290(19): 12247-55.

15. Defilippi P, Di Stefano P, Cabodi S. p130Cas: a versatile scaffold in signaling networks. Trends Cell Biol. 2006; 16(5): 257-63.

16. Chung LY, Tang SJ, Wu YC, et al. Galectin-3 augments tumor initiating property and tumorigenicity of lung cancer through interaction with beta-catenin. Oncotarget. 2015; 6: 4936-52.

17. Chen S, Evans HG, Evans DR. FAM129B/MINERVA, a novel adherens 
junction-associated protein, suppresses apoptosis in HeLa cells. J Biol Chem. 2011; 286: 10201-9.

18. Waisberg J, De Souza Viana L, Affonso Junior RJ, et al. Overexpression of the ITGAV gene is associated with progression and spread of colorectal cancer. Anticancer Res. 2014; 34: 5599-607.

19. Kim YK, Shin DH, Kim KB, et al. MUC5AC and MUC5B enhance the characterization of mucinous adenocarcinomas of the lung and predict poor prognosis. Histopathology. 2015; 67(4): 520-8.

20. Sudo H, Tsuji AB, Sugyo A, et al. AHNAK is highly expressed and plays a key role in cell migration and invasion in mesothelioma. Int J Oncol. 2014; 44: 530-8.

21. Huang J, Wang L, Jiang M, et al. AGR2-mediated lung adenocarcinoma metastasis novel mechanism network through repression with interferon coupling cytoskeleton to steroid metabolism-dependent humoral immune response. Cell Immunol. 2014; 290: 102-6.

22. Liu Z, Zhan Y, Tu Y, et al. PDZ and LIM domain protein 1(PDLIM1)/ CLP36 promotes breast cancer cell migration, invasion and metastasis through interaction with alpha-actinin. Oncogene. 2015; 34: 1300-11.

23. Maftouh M, Belo AI, Avan A, et al. Galectin-4 expression is associated with reduced lymph node metastasis and modulation of Wnt/betacatenin signalling in pancreatic adenocarcinoma. Oncotarget. 2014; 5: 5335-49.

24. Morisaki T, Yashiro M, Kakehashi A, et al. Comparative proteomics analysis of gastric cancer stem cells. PLoS One 2014; 9: e110736.

25. Mardin WA, Petrov KO, Enns A, et al. SERPINB5 and AKAP12 expression and promoter methylation of metastasis suppressor genes in pancreatic ductal adenocarcinoma. BMC Cancer. 2010; 10: 549.
26. Xing AY, Wang YW, Su ZX, et al. Catenin-delta1, negatively regulated by miR-145, promotes tumour aggressiveness in gastric cancer. J Pathol. 2015; 236: 53-64.

27. Tucker GC. Integrins: molecular targets in cancer therapy. CurrOncol Rep. 2006; 8: 96-103.

28. Arumugam T, Deng D, Bover L, et al. New Blocking Antibodies against Novel AGR2-C4.4A Pathway Reduce Growth and Metastasis of Pancreatic Tumors and Increase Survival in Mice. Mol Cancer Ther. 2015; 14: 941-51.

29. Charo IF, Nannizzi L, Smith JW, et al. The vitronectin receptor alpha $\mathrm{v}$ beta 3 binds fibronectin and acts in concert with alpha 5 beta 1 in promoting cellular attachment and spreading on fibronectin. J Cell Biol. 1990; 111(6 Pt 1): 2795-800.

30. Zaidel-Bar R. Job-splitting among integrins. Nat Cell Biol. 2013; 15(6): 575-7.

31. Hynes RO. Integrins: bidirectional, allosteric signaling machines. Cell. 2002; 110(6): 673-87.

32. Madsen CD, Ferraris GM, Andolfo A, et al. uPAR-induced cell adhesion and migration: vitronectin provides the key. J Cell Biol. 2007; 177(5): 927-39.

33. Ferraris GM, Schulte C, Buttiglione V, et al. The interaction between uPAR and vitronectin triggers ligand-independent adhesion signalling by integrins. EMBO J. 2014; 33(21): 2458-72.

34. Smith HW, Marshall CJ. Regulation of cell signalling by uPAR. Nat Rev Mol Cell Biol. 2010; 11(1): 23-36. 